\title{
SMART: A process-oriented methodology for resilient smart cities
}

\author{
Monjur Mourshed \\ School of Engineering, Cardiff University \\ Cardiff, CF24 3AA, United Kingdom \\ mourshedm@ cardiff.ac.uk
}

\author{
Antonio Bucchiarone \\ Fondazione Bruno Kessler \\ 38123 Trento, Italy \\ bucchiarone@fbk.eu
}

\author{
Fahmida Khandokar \\ School of Engineering, Cardiff University \\ Cardiff, CF24 3AA, United Kingdom \\ fkhandokar@gmail.com
}

\begin{abstract}
Cities are engines of economic prosperity and social development. Rapid urbanization and the impacts of climate change have resulted in increased vulnerabilities in cities. On the other hand, the increasing proliferation of connected devices and distributed monitoring of the environment around us has opened up an opportunity to transform the way we create and manage cities. Contextual evidence of performance, outcome and efficiency can now be readily collected at a higher resolution to aid multi-disciplinary and multi-objective decision-making, enabling optimal evolution of cities against the backdrop of constrained resources and intensified vulnerabilities. This paper first argues that distributed and ubiquitous monitoring is at the heart of smart cities. Insights can be inferred from the gathered data with potential for evidence-based decisions at the required spatial and temporal scales. The paper then discusses the development of a comprehensive but concise frameworks called DICES (data, insights, citizen, evidence and standards) for conceptualizing smart cities. The dimensions of DICES are then translated into a process oriented methodology called SMART (specify, monitor, analyze, resolve and transform) by formalizing key aspects of the smart city process. Generality and scalability of DICES and SMART are demonstrated through the development of REPRO, a risk- and evidence-based platform for resilient and optimal design of buildings and infrastructure in a smart city.

Key words-Smart city, resilience, smart city methodology, data-centric approach, SMART, DICES, Adaptable Services.
\end{abstract}

\section{INTRODUCTION}

As a concept, smart city has many definitions depending on the context, goal, application and audience. Most definitions and existing research attempt to explain smart cities from a product-centric viewpoint with a technological underpinning [1], [2]. The application-oriented understanding of smart city technologies has discipline specific bias, often resulting in a lack of interoperability between isolated systems. The evident drawback of siloed applications is that their true potential is seldom realized because of the lack of integration with other systems, which also prevents the consideration of interdependent relationships - both existing and evolving. For example, an intelligent transport system (ITS) that aggregates and fuses in-vehicle data from users with monitored traffic and infrastructure data to provide real-time support for reducing journey time, congestion and fuel use, is a smart mobility solution. On the other hand, the optimal management and dispatching of energy infrastructure assets considering intermittent generations from distributed energy resources (DER) and consumer behavior against dynamic pricing is a smart energy application. Both applications can be designed and implemented on their own but if conceived in an integrated way, the smart energy solution could consider the interdependent effects of electric vehicles (EV) with the electricity grid, as well the use of EV for storing excess generation from DERs, with potential for reducing peak demand for energy a demand side management (DSM) strategy.

Integration among application areas and processes appears to be a strong theme among governments and public bodies for realizing the smart city agenda. The European Commission (EC) has identified eleven priority areas for smart and sustainable cities, categorized into three vertical domains (i.e., application areas) and eight horizontal enabling themes [3], as illustrated in Fig. 1a. Enabling themes are further classified into decisions, insights and funds. The theme, decisions focus on integrating citizens in the decision making process while enabling accelerated policies and regulation, and integrated planning across sectors. Insights, the second set of enabling theme, focuses on the demonstration of performance gains by: benchmarking using metrics and indicators; making use of open data; providing a framework for standards-based development; and sharing knowledge. Despite the name, insights do not adequately highlight the process of gaining insights; i.e., the aggregation and fusion of data, as well as the application of data analytics for inference. The last theme, funds, is European Union (EU) specific, calling for the integration of local solutions in the global and EU markets.

On the oher hand, the UK Government (UK-GOV) has identified five key information-driven aspects of smart cities [4], as shown in Fig. 1c. The basis for information-driven smart strategies is a modern digital infrastructure that allows open access to information as and when needed. An intelligent physical infrastructure makes use of the available data to inform strategic development, while acknowledging the role of citizen centric approaches for improving service delivery. The last two aspects, openness to learn and the transparency of outcomes/performance further highlight the need for an agile, evidence-based approach to smart cities.

The key similarities between the EC and UK-GOV frameworks is that smart cities are essentially information-driven and citizen-centric intelligent built environment and infrastructure. Similar conclusions can be made about most, if not all deliberations on smart cities (for further reading, see [5], [6]). The frameworks also mention gleaning insights from the data, as well as share experiences using metrics and 


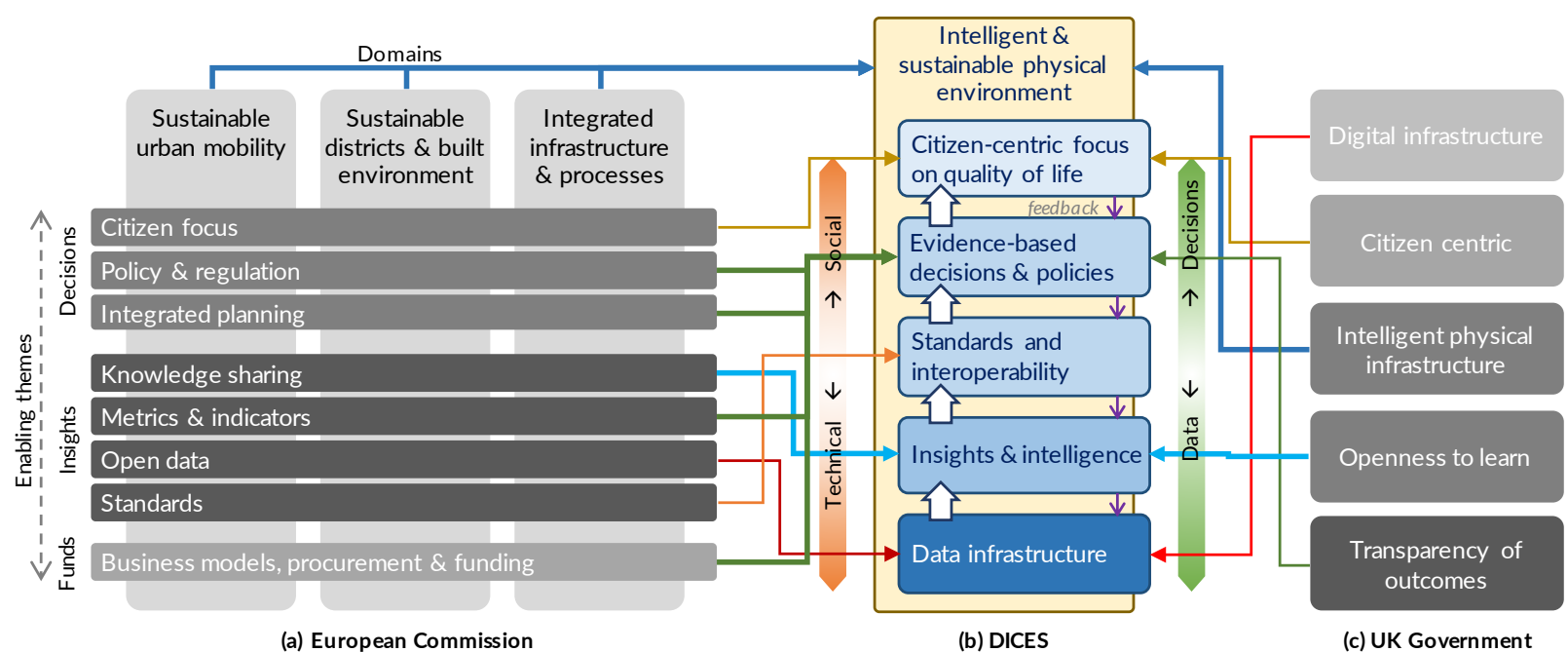

Fig. 1. Mapping between smart city approaches. (a) European Commission's smart and sustainable cities framework, (b) The proposed DICES framework for conceptualizing smart cities, and (c) Key aspects of a smart city as identified by the UK Government.

indicators so that performances can be benchmarked. However, most frameworks, including the two discussed above do not adequately address how data are transformed into insights and then translated to decisions and policies. This is where the discipline specific bias becomes particularly apparent. Inferring insights is mostly computational and typically omitted from frameworks with a socio-organizational bias. The lack of an understanding of the transformation between data, insights and decisions results in an unclear direction about intelligence and resilience - two of the important aspects of the smart concept. There is, therefore, a need for conceptualizing smart cities from a multi-disciplinary perspective so that the interactions between socio-organizational and computational domains are better understood. Moreover, conceptualizing the product and process aspects in a single framework often leads to confusion and room for misinterpretation.

Considering the limitations discussed above, we propose two comprehensive but concise frameworks: DICES - for conceptualizing smart cities from a multidisciplinary perspective, and SMART - for formalizing smart city processes against DICES. The rest of the paper first discusses the frameworks, and then applies them in the context of disaster resilience, an evolving application area requiring the consideration of uncertainty and dynamic system behavior - as an attempt at testing the utility and adaptability of the proposed frameworks in challenging contexts. The paper concludes with a discussion on future research directions.

\section{DICES - A CONCEPTUAL FRAMEWORK}

The overarching requirement of a smart city is its intelligent and sustainable physical environment comprising both the built environment and infrastructures. Intelligence, in the context of smart cities, is the broader ability of the city as a system to: (a) sense its environment, (b) consider multi-disciplinary dependencies for effective modeling of the interacting domains, (c) make optimal decisions, and (d) adapt to changing circumstances such as increasing occurrences of disasters from a changing climate, and changing urban demographics due to aging of the population. Awareness and adaptation are, therefore, key in achieving intelligence (see [7] for additional discussion). Sustainability, as defined here, encompasses not just sustainable development [8] but also the wider holistic aspects. Information is important for achieving an intelligent and sustainable physical environment, and its transformation from data to intelligence requires the inference of insights, which are then translated to evidencebased policies, decisions and regulations with a citizen-centric focus on quality of life. These dimensions are formalized as a conceptual framework called DICES, comprising data, insights, citizen, evidence and standards. Fig. 1b illustrates the DICES framework by mapping elements from EC and UK-GOV themes to demonstrate the comprehensiveness and ability to reconcile the nuances between interpretations.

DICES captures both socio-organizational and computational aspects of a smart city, where data flows from the data infrastructure towards citizen centric decisions; i.e., from technical to social dimensions. The constituent dimensions of DICES are:

- A secure, up-to-date and open digital data infrastructure that enables stakeholders, including citizens access to public and reusable data as and when needed;

- A system for inferring insights and intelligence by applying data analytics on the aggregated heterogeneous data. The system should be as automated as possible for maximizing the potential of the data infrastructure;

- The infrastructures, systems and processes need to be based on standards to foster interoperability between actors and processes. The development and application of standards is a continuous process and informed by feedback from up- and down-stream dimensions;

- Strategies and processes fostering transparent, evidencebased decisions, policies and regulations based on the inferred insights and standards; and 
- A citizen-centric service that minimizes redundancy and maximizes efficiency at all levels of service delivery with the ultimate goal of improving the quality of life of its citizens while not adversely impacting on resources and the environment.

The end product of all five dimensions is an agile and adaptive system that evolves rather than stagnates and learns from experience rather than overlooks evidence.

\section{SMART - A PROCESS-ORIENTED METHODOLOGY}

A closer look at the consolidated DICES framework reveals that the key task in a smart city is the collection and later aggregation of data to support insights and decisions. We, therefore, propose monitoring to be the central task in the proposed SMART (specify, monitor, analyze, resolve and transform) methodology, as illustrated in Fig. 2. Monitoring, in this context is a cyclic process and applies to both the existing and future data infrastructure. It is cyclic in the sense that we continue to update the monitoring (i.e., data) infrastructure by learning from experiences. The remainder of the SMART methodology builds on monitored data and comprises the following process elements:

- Specify: Citizen-centric, sustainable goals and objectives are specified considering the multi- and inter-disciplinary nature of interactions between application sectors. Specifications are often based on an analysis of the previously or existing monitored data;

- Analyze: Success in a data-centric process relies on the ability to glean insights and intelligence from the underlying dataset. This step in the SMART methodology relies on computational, statistical and empirical modeling techniques for inferring insights, some or all of which can be automated to eliminate or reduce obsolescence;

- Resolve: Solution space in a smart city is often multidimensional and decisions are multi-objective. Computational and operational research (OR) techniques are often best suited for multi-dimensional search for optimal decision. Resolve aims to enable evidence-based

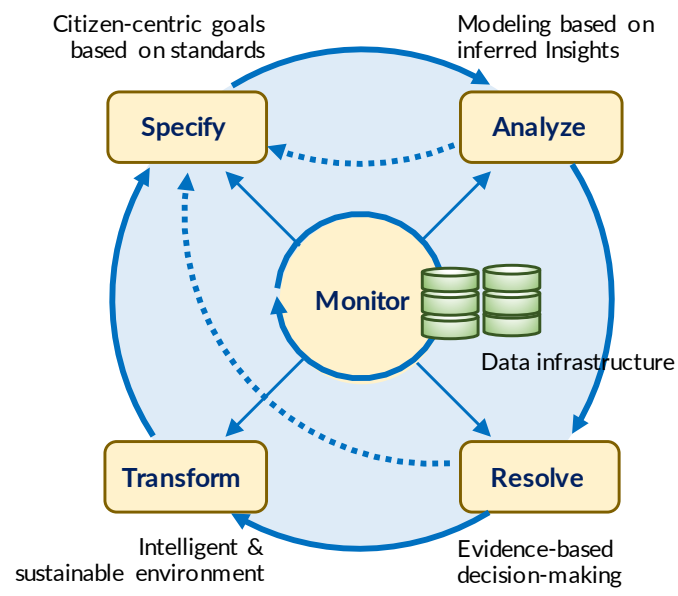

Fig. 2. The SMART process-oriented methodology.
TABLE I

RELATIONSHIP BETWEEN SMART, DICES AND EU SMART CITIES ENABLING THEMES.

\begin{tabular}{|l|l|l|}
\hline SMART & DICES & EU smart cities enabling themes \\
\hline Specify & $\begin{array}{l}\text { Citizen } \\
\text { Evidence }\end{array}$ & $\begin{array}{l}\text { Metrics \& indicators } \\
\text { Citizen focus } \\
\text { Policy \& regulations }\end{array}$ \\
\hline Monitor & $\begin{array}{l}\text { Data infrastructure } \\
\text { Standards }\end{array}$ & $\begin{array}{l}\text { Open data } \\
\text { Standards } \\
\text { Metrics \& indicators }\end{array}$ \\
\hline Analyze & $\begin{array}{l}\text { Insights } \\
\text { Evidence }\end{array}$ & $\begin{array}{l}\text { Integrated planning } \\
\text { Metrics \& indicators }\end{array}$ \\
\hline Resolve & Evidence & Integrated planning \\
\hline Transform & Standards & $\begin{array}{l}\text { Standards } \\
\text { Knowledge sharing }\end{array}$ \\
\hline
\end{tabular}

decisions from insights gathered in the previous step, analyze; however, resolve has feedback loop with specify and analyze which can be taken if necessary; and

- Transform: This step relates to the implementation of the optimal decisions taken at the earlier step and focuses on standards-based evolution of the physical infrastructure so that adaptability and resilience of the smart city is not compromised.

The incorporation of recursive steps such as specify, analyse and resolve in the SMART methodology allows for tweaking to suit specific circumstances or domain-specific processes. The relationship between SMART, DICES and the enabling themes from the EU framework on smart cities and communities are given in Table I. One to many relationships exist between SMART process elements and DICES and the EU framework, primarily due to the fact that SMART is a process oriented methodology while the others are mostly product centric frameworks.

\section{Application of DiCES ANd SMART}

The DICES framework and SMART methodology are designed to maximize their applicability in varying contexts. In order to demonstrate their versatility, the development of a risk-based resilience application is demonstrated here. The application is a design and standards-development platform for risk- and evidence-based design of buildings and infrastructures for adaptation to natural disasters. In the interest of brevity the platform is hereafter referred to as REPRO (Risk- and Evidence-based Platform for Resilient and Optimal Design of Buildings).

\section{A. Background}

The frequency and economic losses from natural disasters have increased significantly in recent years with statistics suggesting an increasing trend of risks associated with disasters [9]. Disaster impacts on built environment are most often complex, primarily due to the interrelationship that exists between buildings, infrastructures, communities, cities and society. Disaster events are often characterized by multiple and/or cascading events leading to economic losses and mortality, and are likely to increase due to anthropogenic climate change 


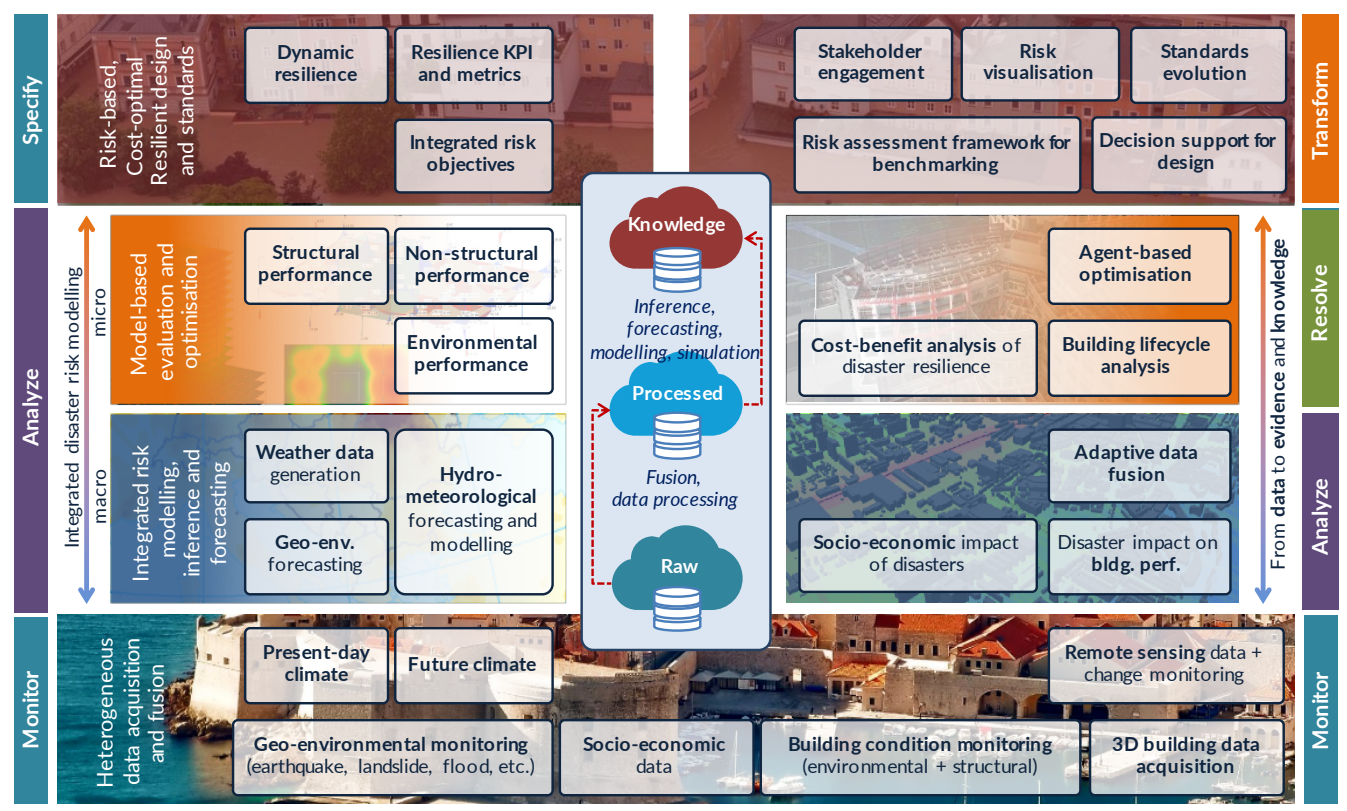

Fig. 3. The REPRO approach and its mapping with the SMART methodology.

[10]. Enhancing resilience in buildings and infrastructure, therefore, requires an integrated approach. Hence, REPRO is designed as a scalable, intelligent, collaborative, interoperable decision support platform for risk-based, cost-optimal resilient design of buildings and critical infrastructure against disaster risks of climate and geological origins.

REPRO takes the view that resilient buildings and infrastructure are an integral part of the smart and resilient cities agenda, and that the risk- and evidence-based understanding and investigation of connectedness and inter-dependence between buildings and infrastructures is the key to a resilient future, both for individual buildings and cities and regions. Monitoring is the basis for activities in REPRO, which leads to the gathering of evidence on disaster risks, event characteristics (magnitude, occurrence and probabilities), impact, and performance of buildings and socio-technical systems. Integrated access to multi-dimensional evidence enables a more accurate inference on performance, based on which design concepts, goals and building standards are defined, which in turn are evaluated and/or optimized until the defined goals have been achieved. Evaluation can be based on domain and/or integrated simulation and modeling of performance (structural and non-structural). Monitored data, when combined with automated inference and learning algorithms, can be used to trigger the process of update or evolution of building standards and designs.

\section{B. Overall approach}

REPRO workflow relies on the proposed SMART methodology involving five steps: specify, monitor, analyze, resolve and transform. The overall REPRO approach is shown in Fig. 3 along with the mapping of the clusters with the SMART process elements. REPRO clustered tasks and layers are integrated via a federated cloud-based computer platform, as illustrated in Fig. 4.

- Heterogeneous data acquisition and fusion addresses one of the key challenges in disaster management, response and resilience related to the lack of availability of data to stakeholders for upstream processing, analysis and informed decision-making. Natural and geological disasters are geo-spatial and temporal in nature, the consideration of the impacts of which on buildings need to be monitored and made accessible so that designers, asset managers and policy makers can react quickly to changing disaster landscape by developing and/or updating design guidelines and building regulations.

- Integrated risk modeling, inference and forecasting makes use of fused and streamed data from heterogeneous sources to infer knowledge of impact (losses due to a disaster), risk (probability of a disaster event) and performance of building systems (damage, degradation) on geospatial and temporal scales. Outputs from integrated risk modeling, inference and forecasting are used as boundary conditions such as current or projected future climates, and flood or earthquake risk maps for further use in: (a) model-based simulation of building design options and standards, and (b) triggering the process of developing new or updating existing guidelines, expected performance ranges (reliability range) and standards.

- Model-based evaluation and optimization - enables multi-domain, integrated evaluations of design and/or standard options over life-cycle stages (design to use) through interoperable, standards-based (IFC-BIM for buildings, CityGML for urban areas and cities) modeling and optimization framework, developed as part of REPRO. Open-standards based integration of software tools allow the use of both proprietary and open-source 


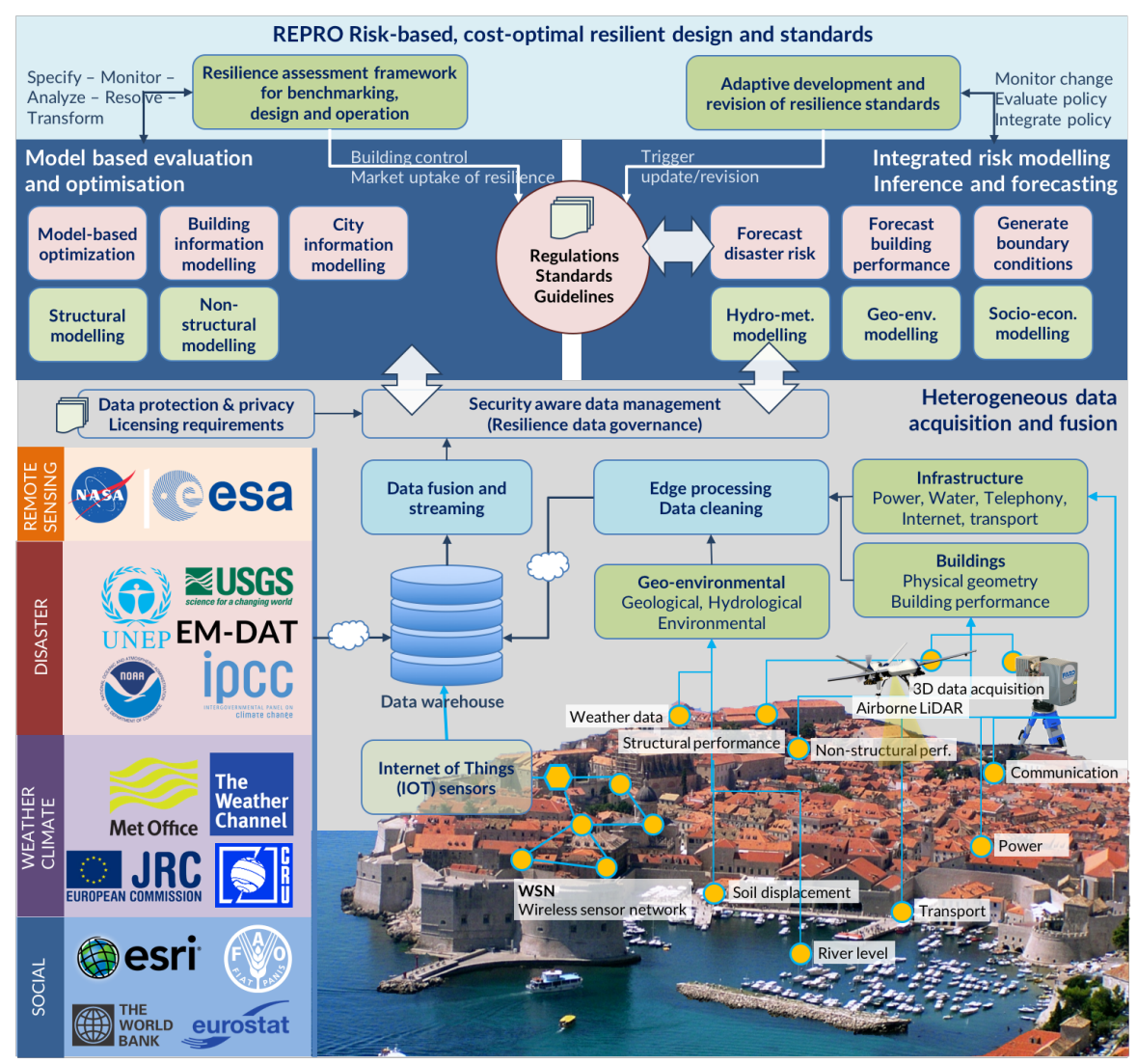

Fig. 4. REPRO platform high-level system architecture.

tools for flexibility. REPRO platform will also allow for integration of inferred knowledge from risk modeling and forecasting using acquired and/or integrated heterogeneous data. The flexibility in REPRO lends itself for wider applications, including recursive and distributed (i.e., holonic) systems for integration between ICT and integrated applications [11].

- Risk-based, cost-optimal resilient design and standards - focuses on the specification of key performance indicators (KPIs), ideally based on consensus. The specified KPIs are then translated to a resilience modeling and scenario-testing framework by taking advantage of the integrated risk modeling and model-based evaluation and optimization tools, enabling the comparison and/or evaluation of multi-objective design/standard goals; e.g. cost vs. resilience and structural vs. non-structural performance. Having access to knowledge and evidence from archived data, REPRO can enable automatic triggering of updates to regulations.

\section{REPRO platform architecture}

The federated cloud-based computer platform in REPRO enables a comprehensive end-user focused decision support for a resilient smart city. The federation is accomplished by factoring in and translating the different functionalities into a set of Web 2.0 services to: (a) streamline the integration of disaster and building performance data from heterogeneous sources, (b) facilitate adaptive data fusion and processing, (c) enable secure, role based access to data by stakeholders and REPRO services and processes, and (d) provide means for interoperable performance evaluation and optimization. The scalability arises from the ability to integrate existing and legacy systems, while enabling the REPRO platform to adapt to future advances in technology. The integration of REPRO services is achieved through REST APIs by implementing on:

- An Application Server that provides a set of uniform resource identifiers (URIs) to access data; and

- A Platform as a Service (PaaS) to accommodate REPRO services that can be called from client applications.

Service-oriented computing can play a key role enabling the integration and interplay between new and legacy city services to solve current and future challenges and support the creation and delivery of innovative and efficient services for client applications. A key challenge that still needs to be overcome for this to become a reality, is the capability of dealing with the continuously changing and complex environment in which smart city applications operate. The system should be resilient to changes in existing services and in client applications requirements, as well as be open and extensible for new functionalities and facilities to become part of the applications. There is therefore the need to develop services that are adaptable 'by design' and to provide a dynamic and scalable runtime environment that makes them resilient to the aforementioned changes. 


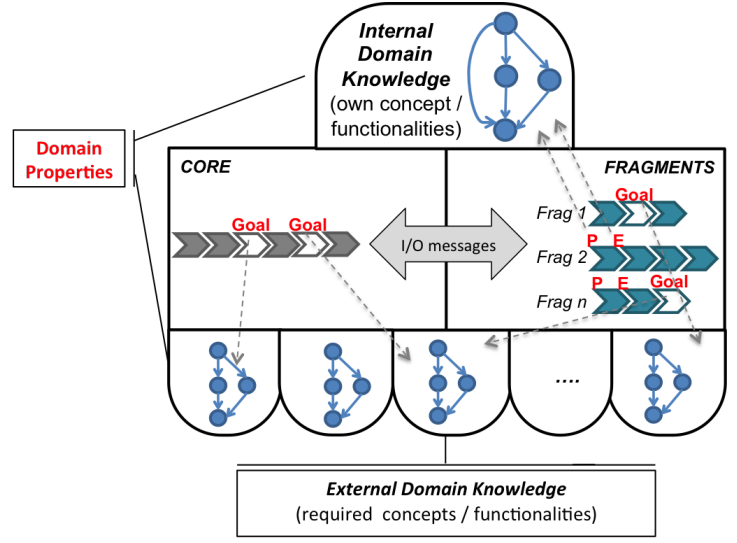

Fig. 5. Domain objects for adaptable by design services.

REPRO client applications are modeled through a set of domain objects (DOs) [12] representing application services as illustrated in Fig. 5. Each DO is characterized by a core process, implementing its own behavior, and a set of fragments, representing the functionalities it provides. Fragments [13] are executable processes that can be received and executed by other DOs. Unlike traditional service-based applications where the behavior of a service is pre-defined, our approach allows the partial specification of the expected operation of DOs through abstract activities that can be refined at runtime, to accomplish a certain goal, according to the fragments offered by other DOs in the application. Due to the dynamic nature of smart city applications, other services are allowed to join the system and offer specific processes at runtime. When client application needs are known, the platform uses suitable DO fragments to specialize its behavior and to provide context-aware functionalities. The dynamic features offered by the platform rely on a set of concepts, describing the operational environment, on which each DO has a partial view. In particular, the internal domain knowledge captures the behavior of the domain concept implemented by the DO, while the external domain knowledge represents domain concepts that are required to accomplish its behavior but for whose implementation it relies on other DOs. Domain knowledge is defined through domain properties, each giving a high-level representation of the concept. Domain properties, modeled as state transition systems, are used to capture the status of the context at a specific time of execution and they evolve as an effect of the execution of a process activity. The resulting adaptive application is a dynamic network of DOs. Potential soft dependencies are established between the DOs in the application. A soft dependency between two DOs becomes a strong dependency if, during the system execution, they interoperate by injecting and executing a fragment.

\section{DISCUSSION AND CONCLUSION}

Contemporary conceptual discussions on smart cities still rely on the understanding from a product perspective, which is inadequate to deal with the complexities and nuances of the process. Having a static product oriented conceptual frame- work hinders the realization of the full potential offered by significant developments in the Internet of Things (IoT), data fusion and analytics, distributed computational intelligence, information modeling and decision science. Through the development of the DICES framework, we demonstrated that key elements of a smart city can be conceptually conceived in a simpler way and that the feedback loops are essential for learning from experiences, as well as for integration between the digital infrastructure and upstream processes.

We then translated the proposed DICES framework into a process-oriented methodology, conveniently called SMART that clusters smart city activities into five atomic steps: specify, monitor, analyze, resolve and transform. Together with the DICES framework, SMART offers flexibility in implementation, demonstrated by applying the methodology in a challenging application domain - the development of a risk- and evidence-based platform for resilient and optimal design of buildings and infrastructure (REPRO) in a smart city. The approach and the architecture of REPRO illustrates the scalability of the SMART approach, as well as the ability to adapt to changing processes and goals. Future work on the topic can be on real implementation and the investigation into generality of the proposed framework and platform.

\section{REFERENCES}

[1] T. Nam and T. A. Pardo, "Conceptualizing smart city with dimensions of technology, people, and institutions," in Proceedings of the 12th Annual International Digital Government Research Conference. New York, NY, USA: ACM, 2011, pp. 282-291.

[2] G. Piro, I. Cianci, L. Grieco, and G. Boggia, "Information centric services in smart cities," Journal of Systems and Software, vol. 88, pp. 169-188, 2014.

[3] EC, European Innovation Partnership on Smart Cities and Communities: Strategic Implementation Plan. Brussels: European Commission, 2013.

[4] BIS, Smart cities: Background paper. London: Department for Business, Innovation \& Skills, 2013.

[5] H. Chourabi, T. Nam, S. Walker, J. R. Gil-Garcia, S. Mellouli, K. Nahon, T. A. Pardo, and H. J. Scholl, "Understanding smart cities: An integrative framework," in 45th Hawaii International Conference on System Science, 2012, pp. 2289-2297.

[6] A. Caragliu, C. D. Bo, and P. Nijkamp, "Smart cities in europe," Journal of Urban Technology, vol. 18, pp. 65-82, 2011.

[7] S. D. Ramchurn, P. Vytelingum, A. Rogers, and N. R. Jennings, "Putting the 'smarts' into the smart grid: A grand challenge for artificial intelligence," Commun. ACM, vol. 55, no. 4, pp. 86-97, 2012.

[8] B. J. Brown, M. E. Hanson, D. M. Liverman, and R. W. Merideth, "Global sustainability: Toward definition," Environmental Management, vol. 11, no. 6, pp. 713-719, 1987.

[9] UNISDR, Building Resilience to Disasters in Europe. Geneva: The United Nations Office for Disaster Risk Reduction, 2014.

[10] IPCC, Climate Change 2014: Impacts, Adaptation, and Vulnerability. Geneva: Intergovernmental Panel on Climate Change, 2014.

[11] M. Mourshed, S. Robert, A. Ranalli, T. Messervey, D. Reforgiato, R. Contreau, A. Becue, K. Quinn, Y. Rezgui, and Z. Lennard, "Smart grid futures: Perspectives on the integration of energy and ICT services," Energy Procedia, vol. 75, pp. 1132 - 1137, 2015.

[12] A. Bucchiarone, M. de Sanctis, A. Marconi, M. Pistore, and P. Traverso, "Design for adaptation of distributed service-based systems," in ICSOC, 2015, pp. 383-393.

[13] A. Sirbu, A. Marconi, M. Pistore, H. Eberle, F. Leymann, and T. Unger, "Dynamic composition of pervasive process fragments," in ICWS"11, 2011, pp. 73-80. 